Obere Extremität $2011 \cdot 6: 157-158$

DOI 10.1007/s11678-011-0125-1

Online publiziert: 17. August 2011

(c) Springer-Verlag 2011

\author{
R. Hierner \\ Plastische, Rekonstruktive, Ästhetische und Handchirurgie, \\ Universität Duisburg-Essen, Universitätsklinikum Essen
}

\title{
Vaskularisierte Knochentransplantate im Bereich der oberen Extremität
}

Ollier [8] gab dem Konzept, Knochendefekte und schlecht heilende Frakturen durch Knochentransplantate zu sanieren, im Jahr 1867 erstmalig eine wissenschaftliche Basis. Der Schlüssel für eine erfolgreiche Knochendefektdeckung liegt in der Vaskularisierung des Knochentransplantats. Bei vaskularisierten Knochentransplantaten wird die Durchblutung nicht (gefäßgestielte Transposition) oder nur kurzzeitig (freie mikrovaskuläre Knochentransplantation) unterbrochen. Konventionelle, nichtvaskularisierte Knochentransplantate sind aufgrund der Unterbrechung ihrer Blutversorgung ohne ein ersatzstarkes Lager nicht mehr in der Lage, weiterzuwachsen. Nur wenige Osteozyten unterhalb des Periostes überleben durch Diffusion, die anderen werden nekrotisch. Somit hat das konventionelle, nichtvaskularisierte Transplantat primär nur eine Überbrückungsfunktion. Barth [1] postulierte 1895, dass das Transplantat erst durch schleichenden Ersatz nach erfolgter Neovaskularisierung aus dem Transplantatlager und den beiden Knochenstümpfen umgebaut werden kann. Im Gegensatz zum nichtvaskularisierten Knochentransplantat heilt das vaskularisierte wie eine Etagenfraktur im Empfängergebiet ein. Da das Transplantat seine eigene, völlig ausreichende Vaskularisierung mit in das Empfängerlager bringt, ist der Anteil der Neovaskularisierung aus dem Transplantatlager viel geringer. Die entstehenden vaskulären Verbindungen zwischen Transplantat und Empfängerlager können als zusätzliche Versorgung und Leitschiene für den ortsgerechten Einbau gedeutet werden.
Die Technik der vaskularisierten Knochentransplantation wurde bereits am Ende des 19. Jahrhunderts eingeführt. Um die Blutversorgung transplantierter Knochen zu erforschen, untersuchten Hellstadius in den 1940er Jahren [5] sowie Davis und Taylor Anfang der 1950er Jahre [2] das biologische Verhalten von muskelgestielten Knochentransponaten. Andere Autoren untersuchten die gefäßgestielten Knochentransponate. Die klinische Anwendung gestielter Knochentransplantate (Knochentransponate) war jedoch durch die geringe Menge an transponierbarem Knochen und die räumliche Einschränkung des Schwenkradius begrenzt. Ihr Einsatz erfolgte hauptsächlich in der Mund-, Kiefer- und Gesichtschirurgie und als Hahn-Brandes-Operation bei der Behandlung von Tibiadefekten in der Kinderorthopädie. Nachdem Strauch et al. [10], Haw et al. [4], O'Brien [7] sowie Östrup und Frederickson [9] in den 1970er Jahren erfolgreich Knochen mit dessen zu- und abführenden Blutgefäßen frei mit mikrochirurgischen Techniken bei Tieren transplantiert hatten, berichteten McCullogh und Frederickson [6] im Jahr 1973 von der ersten freien vaskularisierten Rippentransplantation am Menschen. In der Folgezeit wurden zahlreiche weitere vaskularisierte Knochentransplantate beschrieben und eingesetzt. Nach der anfänglichen Euphorie, mit der neuen Technik nun alle Knochendefekte zuverlässig decken zu können, zeigten Nachuntersuchungen größerer Serien, dass auch die vaskularisierte Knochentransplantation von einem möglichst ersatzstarken Lager profitiert. Kompromis- se beim Débridement oder anderen lagerverbessernden Eingriffen führen zu deutlich schlechteren Einheilungsraten.

Die vaskularisierte Knochentransplantation ist eine Möglichkeit der Therapie von Knochendefektzuständen, nicht die einzige. Die konventionelle, nichtvaskularisierte Knochentransplantation und die Kallusdistraktion sind weitere Therapiemöglichkeiten. Es ist wichtig, die Therapieoptionen nicht als konkurrierende, sondern sich ergänzende Möglichkeiten zu sehen.

Wir hoffen, dem Leser mit diesem Themenheft einen guten Überblick über die Möglichkeiten und Grenzen der vaskularisierten Knochentransplantation im Bereich der oberen Extremität an die Hand zu geben.

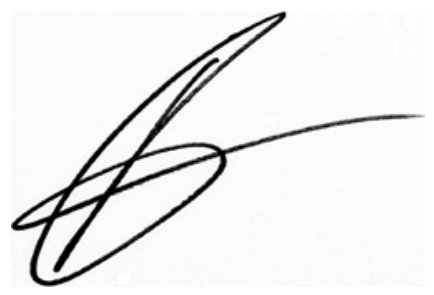

Prof. Dr. med. R. Hierner

\section{Korrespondenzadresse \\ Prof. Dr. R. Hierner}

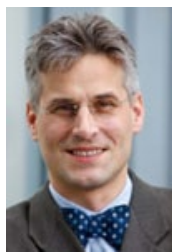

Plastische, Rekonstruktive,

Ästhetische und Handchirurgie, Universität Duisburg-Essen, Universitätsklinikum Essen Hufelandstrasse 55,

45147 Essen

robert.hierner@uk-essen.de 


\section{Literatur}

1. Barth A (1895) Histologische Untersuchungen über Knochentransplantationen. Beitr Pathol Anat 17:65-142

2. Davis JB, Taylor AN (1952) Muscle pedicle bone grafts: Experimental Study. Arch Surg. 65:330

3. Hahn E (1884) Eine Methode Pseudarthrose der Tibia mit großem Knochendefekt zur Heilung zu bringen. Zentr Chir 11:337-341

4. Haw CS, O'Brien B, Kurata T (1978) The microsurgical revascularization of resected segments of tibia in the dog. J Bone Jt Surg 60B:266-269

5. Hellstadius A (1942) On the ability of bone tissue (incl."os novum") to survive in pedicled bone grafts. Acta Chir Scand 86:85-109

6. McCullogh DW, Frederickson JM (1973) Neovascularized rib graft to reconstruct mandibular defects. Can J Otolaryngol 2:96

7. O'Brien B (1977) Microvascular reconstructive surgery. Churchill Livingstone, Edinburgh, London, New York

8. Ollier L (1867) Traité expérimental et Clinique de la régénération des os et de la production artificielle du tissu osseux. Masson, Paris

9. Östrup LT, Frederickson IM (1974) Distant transfer of a free, living bone graft by microvascular anastomoses: An experimental study. Plast Reconstr Surg 54:274-285

10. Strauch B, Bloomberg AE, Lewin ML (1971) An experimental approach to mandibula replacement: Island vascular composite rib grafts. Br J Plast Surg 24:334-341

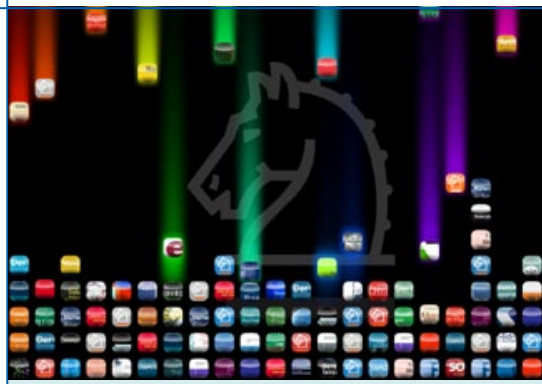

\section{Springer Realtime}

Ein schneller Überblick, was gerade weltweit gelesen wird

Springer hat mit der Website realtime. springer.com ein neues kostenloses Tool geschaffen, mit dem in Echtzeit verfolgt werden kann, welche Zeitschriften- und Buchinhalte, Bilder oder Protokolle weltweit von den Springer-Plattformen SpringerLink, Springerlmages und SpringerProtocols heruntergeladen werden. Ziel ist, den Ärzten und Wissenschaftlern nützliche Informationen darüber zu geben, wie die Literatur genutzt wird.

Folgende Menüpunkte stehen zur Verfügung:

\section{KEYWORDS}

Hier werden die häufigsten Schlüsselbegriffe der letzten 200 heruntergeladenen Artikel in einer "Tag Cloud" dargestellt. Diese wird alle 10 s aktualisiert.

\section{MAP}

Unter diesem Punkt erscheint auf einer Weltkarte genau dort ein Symbol, wo gerade ein Download von einer SpringerOnline-Plattform erfolgt.

\section{FEED}

Diese Darstellung listet die jüngsten Downloads auf. Ein Klick auf das Coversymbol führt zur Internetseite der Publikation. Auf dieser Seite kann man die Anzahl erfolgter Downloads und weitere Infos einsehen.

\section{ICON}

Jeder aktuelle Download eines SpringerInhaltes ist in Form eines herabfallenden Symbols in Echtzeit dargestellt. Klickt man auf eines der Symbole, erscheint die Publikationsquelle und ein Link zu deren Realtime-Internetseite. 